\title{
FHL2 inhibits the activated osteoclast in a TRAF6-dependent manner
}

\author{
Shuting Bai, ${ }^{1}$ Hideki Kitaura, ${ }^{1}$ Haibo Zhao, ${ }^{1}$ Ju Chen, ${ }^{2}$ Judith M. Müller, ${ }^{3}$ Roland Schüle, ${ }^{3}$ \\ Bryant Darnay, ${ }^{4}$ Deborah V. Novack, ${ }^{1,5}$ F. Patrick Ross, ${ }^{1}$ and Steven L. Teitelbaum ${ }^{1}$

\begin{abstract}
1Department of Pathology, Washington University School of Medicine, St. Louis, Missouri, USA. 2Department of Medicine, School of Medicine, University of California at San Diego, La Jolla, California, USA. 3Universitäts-Frauenklinik und Zentrum für Klinische Forschung, Klinikum der Universität Freiburg, Freiburg, Germany. ${ }^{4}$ Department of Experimental Therapeutics, The University of Texas, MD Anderson Cancer Center, Houston, Texas, USA. ${ }^{5}$ Department of Medicine, Washington University School of Medicine, St. Louis, Missouri, USA.
\end{abstract}

\begin{abstract}
TNF receptor-associated factor 6 (TRAF6) associates with the cytoplasmic domain of receptor activator of NF- $\kappa B$ (RANK). This event is central to normal osteoclastogenesis. We discovered that TRAF6 also interacts with FHL2 (four and a half LIM domain 2), a LIM domain-only protein that functions as a transcriptional coactivator or corepressor in a cell-type-specific manner. FHL2 mRNA and protein are undetectable in marrow macrophages and increase pari pass $u$ with osteoclast differentiation in vitro. FHL2 inhibits TRAF6-induced NF- $\kappa B$ activity in wild-type osteoclast precursors and, in keeping with its role as a suppressor of TRAF6-mediated RANK signaling, TRAF6/RANK association is enhanced in FHL2 ${ }^{-/}$osteoclasts. FHL2 overexpression delays RANK ligand-induced (RANKL-induced) osteoclast formation and cytoskeletal organization. Interestingly, osteoclast-residing FHL2 is not detectable in naive wild-type mice, in vivo, but is abundant in those treated with RANKL and following induction of inflammatory arthritis. Reflecting increased RANKL sensitivity, osteoclasts generated from $\mathrm{FHL2}^{-/-}$mice reach maturation and optimally organize their cytoskeleton earlier than their wild-type counterparts. As a consequence, $\mathrm{FHL2}^{-/-}$osteoclasts are hyperresorptive, and mice lacking the protein undergo enhanced RANKL and inflammatory arthritis-stimulated bone loss. FHL2 is, therefore, an antiosteoclastogenic molecule exerting its effect by attenuating TRAF6-mediated RANK signaling.
\end{abstract}

\section{Introduction}

Osteoclasts are polykaryons unique in their capacity to resorb bone (1). They are derived from monocyte/macrophage precursors that assume the osteoclast phenotype under the aegis of the key osteoclastogenic cytokine, receptor activator of NF-KB (RANK) ligand (RANKL) (2). RANKL and its receptor RANK (3) are respective members of the TNF superfamilies of cytokines and receptors, but RANKL enjoys structural features that endow it with its osteoclastogenic properties (4).

RANK, like other members of the TNF family of receptors, mediates its intracellular signals via a group of proteins known as TNF receptor-associated factors (TRAFs) (5). While a number of TRAFs are promiscuous in associating with TNF family receptors, only RANK and CD40 recognize and signal through TRAF6 $(6,7)$. The fact that TRAF6 plays an essential role in osteoclastogenesis is established by the presence of severe osteopetrosis in mice deleted of the adaptor protein $(8,9)$. Thus, delineation of the bone resorptive process requires an understanding of the means by which TRAF6 mediates RANK-induced osteoclastogenesis.

The LIM domain represents a protein motif characterized by double zinc fingers (10). It is a component of an array of molecules, many of which serve as adapters and modifiers of proteinprotein interactions. The four and a half LIM domain (FHL) sub-

Nonstandard abbreviations used: ACT, activator of CREM in testis; BMM, bone marrow macrophage; CREM, cAMP response element modulator protein; FHL, four and a half LIM domain; RANK, receptor activator of NF-KB; RANKL, RANK ligand; TRACP $5 b$, tartrate-resistant acid phosphatase $5 b$; TRAF, TNF receptor-associated factor; TRAP, tartrate-resistant acid phosphatase.

Conflict of interest: The authors have declared that no conflict of interest exists.

Citation for this article: J. Clin. Invest. 115:2742-2751 (2005).

doi:10.1172/JCI24921. family of these proteins consist of 5 members, FHL1, $-2,-3$, and -4 and activator of CREM (cAMP response element modulator protein) in testis (ACT), each consisting exclusively of LIM domains (11). FHL proteins are not known to be expressed in bone cells or participate in skeletal function.

In our exploration of the means by which TRAF6 mediates osteoclastogenesis, we found it associates with FHL2, which is expressed only in activated osteoclasts in vivo. Furthermore, FHL2, by displacing TRAF6 from RANK, attenuates osteoclast formation and cytoskeletal organization via inhibition of NF- $\mathrm{KB}$. As a consequence, osteoclastogenesis and bone resorption are accelerated in the face of FHL2 deficiency. FHL2 therefore represents a physiological regulator of stimulated osteoclast differentiation and skeletal mass.

\section{Results}

TRAF proteins interact with FHL2. We initially utilized TRAF4 as bait in a yeast 2-hybrid system. A mouse embryo cDNA library was screened and FHL2 revealed itself as an interesting target (not shown). To determine whether FHL2 also interacts with TRAFs in mammalian cells, we expressed FLAG-tagged TRAF2, TRAF4, and TRAF6 and HA-tagged FHL2 in 293 T cells. The cells were lysed and the lysate was immunoprecipitated with anti-FLAG antibody, then subjected to Western blot with anti-HA antibody. To assess relative expression of the transfected plasmids, anti-FLAG and anti-HA immunoblots were generated from equal amounts of lysates. As seen in Figure 1A, each FLAG-tagged TRAF associates with FHL2-HA, but TRAF6 does so most effectively, particularly in light of its abundance relative to TRAFs 2 and 4.

Since TRAF6 is a crucial RANKL/RANK signaling mediator (12), we turned our attention to its predominant association with FHL2 with the hypothesis that the latter may regulate osteoclast function. 


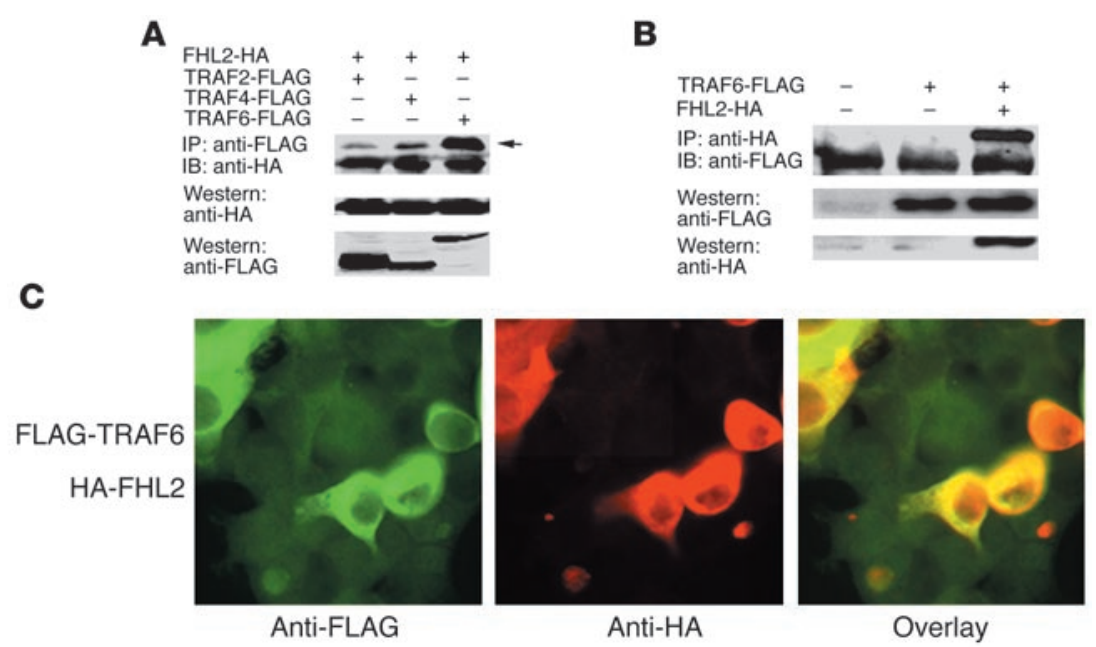

Figure 1

TRAF proteins interact with FHL2. (A) FLAG-tagged TRAF2, TRAF4, and TRAF6 and HAtagged FHL2 were cotransfected into 293T cells. Protein expression level was detected by immunoblot with anti-FLAG and anti-HA antibodies. Cell lysates were immunoprecipitated with anti-FLAG antibody and the immunoprecipitates immunoblotted with anti-HA antibody. A specific band appears in all TRAF-containing immunoprecipitates (arrow). (B) HAtagged FHL2 and FLAG-tagged TRAF6 were cotransfected into 293T cells. Anti-HA antibody was used for immunoprecipitation and anti-FLAG antibody for immunoblotting. (C) The same cells described in B were subjected to immunofluorescent microscopy using an anti-FLAG rabbit polyclonal antibody and FITC-labeled secondary antibody (green reaction product) or anti-HA monoclonal antibody and TRITC-labled secondary antibody (red reaction product). FHL2/TRAF6 colocalization was documented by simultaneous exposure yielding the yellow reaction product (overlay). Magnification: $\times 200$.

To confirm the interaction between the 2 signaling molecules, we once again used 293 T cells transfected with TRAF6-FLAG and FHL2-HA, in this instance immunoprecipitating HA and immunoblotting FLAG. Once again, FHL2 coimmunoprecipitates with TRAF6 (Figure 1B). Furthermore, the intracellular association of TRAF6 and FHL2, when overexpressed in $293 \mathrm{~T}$ cells, is evident by immunofluorescent microscopy (Figure 1C).

All domains of FHL2 are required to interact with TRAF6. Various combinations of LIM domains of FHL2 are required to associate with individual binding partners. For example, deletion of any FHL2 LIM domain, including its half $\mathrm{N}$ terminal moiety, obviates association with the $\beta 1 \mathrm{~A}$ integrin subunit (13). To detail the components of FHL2 that mediate its recognition of TRAF6, we individually deleted each of its LIM domains and transfected the constructs, as HA-tagged proteins, into $293 \mathrm{~T}$ cells. In each circumstance, the cells were also transfected with TRAF6-FLAG (Figure 2A). Anti-HA immunoprecipitates were immunoblotted with anti-FLAG antibody. Once again, the full-length proteins interact, but deletion of any complete LIM domain or the N terminal half domain of FHL2 ablates this association. We then turned to the domain(s) of TRAF6 required to interact with FHL2. Thus, we transfected 293T cells with FLAG-tagged full-length TRAF6 or NH2 terminal deletion constructs lacking the ring domain (TRAF6-NR) or containing only the TRAF domain (TRAF6C). As seen in Figure 2B, the products of each TRAF6 construct immunoprecipitate with FHL2. Thus, the TRAF domain of TRAF6 suffices for its recognition of FHL2.

FHL2 is specifically expressed during osteoclast differentiation in vitro. We next asked if FHL2 is expressed in authentic osteoclasts in vitro. To this end, we cultured WT and FHL2-/- bone marrow macrophages (BMMs) in M-CSF and RANKL. Total RNA was extracted on days 0,2 , and 4 and RT-PCR performed to detect FHL2 mRNA. While FHL2 mRNA was nondetectable in BMMs, its expression was incrementally enhanced in WT cells as they committed to the osteoclast phenotype (Figure 3A). Similarly, FHL2 protein appears with osteoclast differentiation (Figure 3B). As expected, neither FHL2 message nor protein is demonstrable in osteoclasts lacking the gene. Establishing specificity, FHL1, FHL4, and ACT mRNAs are undetectable in BMMs and osteoclasts while FHL3 mRNA is constitutively expressed throughout the process but not induced by RANKL and M-CSF (Figure 3C).

Endogenous FHL2 and TRAF6 interact in osteoclasts. Having discovered that overexpressed FHL2 and TRAF6 interact in transformed cells, we asked if the same endogenous proteins associate in authentic osteoclasts, and if so, whether short-term RANKL stimulation has an impact on the phenomenon. Thus, BMMs or osteoclasts were serum- and cytokine-starved for 3 hours and then treated with RANKL or vehicle for 30 minutes. Immunoblotting of TRAF6 immunoprecipitates with anti-FHL2 antibody establishes that the 2 proteins associate in osteoclasts in a manner not impaired by acute RANKL exposure (Figure 4A). The intracellular association of TRAF6 and FHL2 is buttressed by their colocalization in mature osteoclasts as documented by immunofluorescent microscopy (Figure 4B).

FHL2 blocks RANK / TRAF6 association. The fact that FHL2 associated with TRAF6 raised the possibility that formation of this complex on RANK blunts RANK/TRAF6 signaling. We tested this concept by first establishing that both TRAF6 (Figure 5A) and FHL2 (Figure 5B) associate with RANK when overexpressed in 293 T cells. TRAF6/RANK recognition is enhanced in the absence of FHL2 in authentic preosteoclasts, indicating that the LIM domain protein may blunt osteoclastogenic signals (Figure 5C).

FHL2 modulates NF- $\kappa B$ activity. Its association with TRAF6 in osteoclasts suggests FHL2 may participate in osteoclast function. Because the RANK/TRAF6 complex signals via NF-кB, we asked whether FHL2 has an impact on this transcription complex. To determine the impact of FHL2 on NF-кB in authentic osteoclasts, we turned to nuclear translocation of NF- $\kappa \mathrm{B}$, a requisite event in its transcriptional activity. WT or FHL2/- BMMs were exposed to RANKL and M-CSF for 3 days, starved for 3 hours, and once again exposed to RANKL. Nuclear proteins were extracted, at 0 , 5,15 , and 30 minutes, and immunoblot was performed with an antibody to the p 65 NF- $\kappa$ B subunit. As seen in Figure 6A, RANKL treatment induces more rapid and profound p 65 nuclear translocation in FHL2 $2^{-/-}$than in WT cells.

To determine the transcriptional consequences of FHL2-restricted NF- $\kappa B$ activation, we assessed RANKL stimulation of selective $\mathrm{NF}-\kappa \mathrm{B}-$ responsive genes in WT and FHL2-deleted preosteoclasts (Figure $6 \mathrm{~B})$. In fact, RANKL-induced expression of $I \kappa B \alpha$ and TNF- $\alpha$ in $\mathrm{mRNA}$, both of which are the products of NF- $\mathrm{KB}$ target genes, is enhanced in the cells lacking the LIM protein. On the other 
A

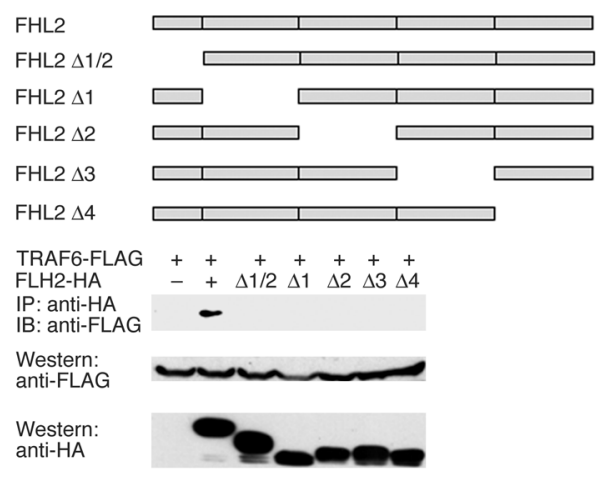

B
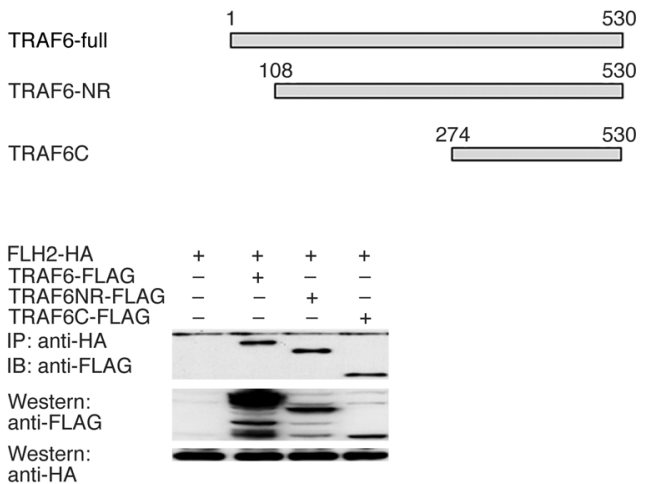

Figure 2

FHL2 and TRAF6 domains required for coassociation. (A) FHL2 deletion constructs were generated by mutagenesis of full-length FHL2. FLAG-tagged TRAF6 and HA-tagged FHL2 constructs were cotransfected into 293T cells. Anti-HA antibody was used for immunoprecipitation and anti-FLAG antibody for immunoblotting. Deletion of any LIM domain of FHL2 abolishes its interaction with TRAF6. The diagram defines $\Delta$ mutants. (B) TRAF6 constructs were generated by PCR. FLAG-tagged full-length TRAF6 (TRAF6-full), ring domain-deleted TRAF6 (TRAF6-NR), and the complete TRAF domain (TRAF6C) were cotransfected with HA-tagged FHL2 into 293T cells. Anti-HA antibody was used for immunoprecipitation and anti-FLAG antibody for immunoblotting.

hand, TNF- $\alpha$, which, like RANKL, is a TNF superfamily member, fails to substantially distinguish p 65 translocation in FHL2 $2^{-/-}$preosteoclasts relative to WT, establishing specificity of RANKL in the FHL2 osteoclastogenic signaling pathway (Figure 6C).

Finally, we reasoned that if its absence enhances NF- $\mathrm{BB}$ signaling, the opposite should obtain in osteoclasts overexpressing FHL2. To test this hypothesis, we retrovirally transduced WT BMMs with an FHL2 expression plasmid or empty vector. The cells were treated with M-CSF plus increasing amounts of RANKL for 4 days and then assayed for RANKL-dependent $\mathrm{NF}-\kappa \mathrm{B}$ activity. In keeping with a central role for the transcription complex in TRAF6-stimulated osteoclast formation and function, overexpressed FHL2 arrests RANKL-induced I $\mathrm{B} \alpha$ phosphorylation (Figure 6D).

Absence of FHL2 accelerates osteoclast differentiation and cytoskeletal organization. These data indicate that FHL2 may delay osteoclast recruitment and/or function. To determine if this is so, we treated FHL2 ${ }^{-/-}$and WT BMMs with increasing doses of RANKL and M-CSF. After 4 days, the number of characteristic, well-spread osteoclasts was substantially greater in cultures derived from FHL2 $2^{-/-}$mice than in those from WT mice (Figure 7, A and B). The enhancement of osteoclast size and spreading is particularly evident at lower concentrations of RANKL, indicating that FHL2 deficiency sensitizes osteoclasts to the cytokine.The accelerated rate of FHL2 $2^{-/-}$osteoclast precursor differentiation is substantiated by increased expression of the 2 osteoclastogenic markers, NFAT2 and cathepsin K (Figure 7C).

The propensity of FHL2-/- osteoclasts to spread suggests enhanced organization of their cytoskeleton. To determine if this is so, we generated osteoclasts on dentin. Similarly to their appearance on plastic (Figure 7A), FHL2-deficient osteoclasts on the resorptive substrate are larger than WT and have more pronounced actin rings, a critical hallmark of cytoskeletal organization (Figure 7D).

Overexpression of FHL2 attenuates osteoclast differentiation and spreading. Given our findings that FHL2 deletion accelerates osteoclast differentiation and cytoskeletal organization, we predicted that overexpression of the protein would exert the opposite effect, namely, blunted maturation and spreading of bone resorptive cells. As shown in Figure 8, control BMMs exposed to $50 \mathrm{ng} / \mathrm{ml}$ RANKL but not those overexpressing FHL2 differentiate into characteristic osteoclasts. At $100 \mathrm{ng} / \mathrm{ml}$ RANKL, the FHL2-transduced cells begin to undergo osteoclastogenesis, but the polykaryons failed to assume the spread configuration apparent in cells bearing empty vector. Spreading of FHL2-overexpressing osteoclasts is evident in cultures containing $200 \mathrm{ng} / \mathrm{ml}$ RANKL, but even in this circumstance, many cells remain unspread and the characteristic osteoclasts are smaller than control.

FHL2-deficient osteoclasts undergo early apoptosis. The osteoclast is a terminally differentiated cell and as such enjoys a defined life span. This being the case, one would predict that cells reaching terminal differentiation rapidly would also initiate early apoptosis. In fact, by day 4 of culture, programmed cell death is increased more than 2 -fold in osteoclasts lacking FHL2 (Figure 9A). In keeping with this observation, caspase 3 is activated earlier during the osteoclastogenic process in $\mathrm{FHL2}^{-/-}$as compared with WT cells (Figure 9B).

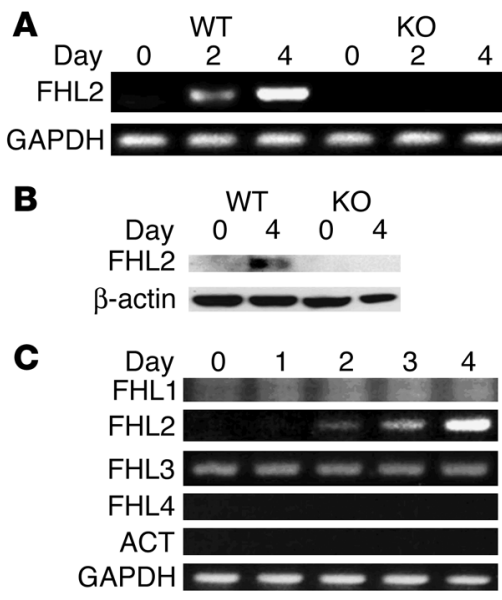

Figure 3

FHL2 is induced during osteoclastogenesis. (A) WT and $\mathrm{FHL}^{-/-}$(KO) BMMs were cultured in M-CSF and RANKL for up to 4 days. FHL2 mRNA expression was determined by RT-PCR. GAPDH mRNA served as loading control. (B) BMMs and osteoclasts, treated as in A, were lysed, and FHL2 expression was determined by immunoblot. $\beta$-actin served as loading control. (C) BMMs were treated with M-CSF and RANKL for up to 4 days, and FHL family member mRNAs were determined by RT-PCR. 
A

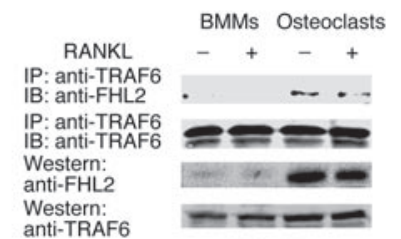

B

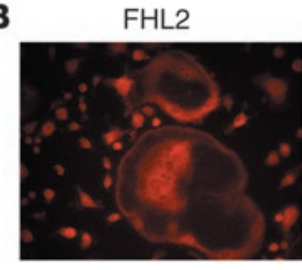

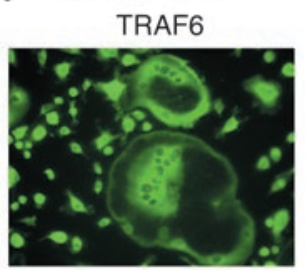

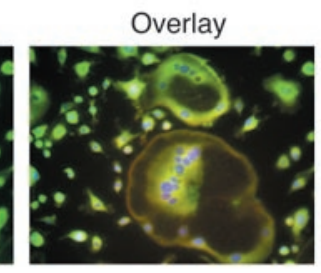

\section{Figure 4}

FHL2 interacts with TRAF6 in osteoclasts. (A) WT BMMs and day 4 osteoclasts were starved for 3 hours and maintained with and without RANKL (100 ng/ml) for 30 minutes. TRAF6 was immunoprecipitated from lysates and the immunoprecipitate blotted with anti-FHL2 or anti-TRAF6 antibodies. Lysate content of the 2 proteins was determined by immunoblot. (B) Mature osteoclasts (day 5) were subjected to immunofluorescent microscopy using an anti-TRAF6 rabbit polyclonal antibody and FITC-labeled secondary antibody (green reaction product) or anti-FHL2 monoclonal antibody and TRITC-labled secondary antibody (red reaction product). FHL2/TRAF6 colocalization was documented by simultaneous exposure yielding the yellow reaction product (overlay). Magnification: $\times 200$.
Absence of FHL2 enhances osteoclast resorptive activity. To determine whether acceleration of differentiation and cytoskeletal organization of $\mathrm{FHL}^{-/-}$osteoclasts extends to the cells' capacity to degrade bone, we generated osteoclasts from mutant and WT BMMs, on dentin, for 4 days. Figure 10, A and B, shows that resorptive lacuna formation by FHL2-deficient osteoclasts during this period is enhanced approximately 10 -fold relative to that in WT.

These data substantiate stimulated net resorptive activity in the absence of FHL2 in vitro. Together with the fact that RANKL induces FHL2 expression by osteoclasts in vivo, they also suggest that FHL2-deficient mice, treated with the cytokine, would resorb bone more aggressively than their WT counterparts. In fact, tartrate-resistant acid phosphatase 5b (TRACP 5b) serum levels of FHL2-/- mice treated with RANKL for 7 days are increased 4-fold relative to those of WT (Figure 10C). Reflecting this enhanced resorptive activity, trabecular bone volume of RANKL-treated mutant mice is approximately one-third that of their WT counterparts (Figure 10D). On the other hand, there is no significant difference in bone mass between PBS-injected WT and mutant mice.

FHL2 is expressed only in activated osteoclasts in vivo. The lack of significant change in bone mass in PBS-injected FHL2 $2^{-/-}$mice challenges the relevance of the protein in basal osteoclast function. To address this issue, we assessed FHL2 expression by osteoclasts in vivo. In fact, osteoclasts in naive WT mice do not contain detectable immunoreactive protein (data not shown). Because osteoclasts lacking NF- $\mathrm{KB}$-inducing kinase, when maintained in culture, reflect the activated and not basal state of their in vivo counterparts (14), we asked whether the same obtain regarding $\mathrm{FHL2}^{-/-}$bone resorptive cells. Hence we injected soluble RANKL $(100 \mu \mathrm{g} /$ day $)$ or PBS for 7 days into WT and $\mathrm{FHL2}^{-{ }^{-}}$mice and once again immunostained histological sections with anti-FHL2 mAb. Similarly to naive WT mice, those receiving $\mathrm{PBS}$ contain no osteoclast-residing immunoreactive FHL2 (Figure 11A). In contrast, numerous FHL2-expressing osteoclasts are present in the RANKL-treated animals. No immunostaining is detectable in megakaryocytes, the bones of FHL2 $2^{-/-}$mice, or second antibody-exposed sections of RANKLtreated animals (not shown).

The fact that FHL2 is restricted to activated osteoclasts, in vivo, suggests that expression of the protein by these cells may also be abundant in diseases of accelerated bone resorption. We therefore generated a murine model of inflammatory arthritis, which, like that affecting humans, is characterized by peri- articular osteolysis (15). As seen in Figure 11B, joint-residing osteoclasts in mice with inflammatory arthritis also contain an abundance of immunoreactive FHL2. Documenting that absence of FHL2 enhances bone resorption in the context of this disease, serum TRACP $5 \mathrm{~b}$ levels are substantially greater in FHL2 $2^{-1-}$ than in WT arthritic mice (Figure $11 \mathrm{C}$ ). On the other hand, FHL2 does not impact the numbers of osteoclasts in naive mice and those treated with RANKL or afflicted with inflammatory arthritis (data not shown). Consistent with cytoskeletal changes, therefore, the accelerated bone resorption in the mutant animals reflect stimulated osteoclast activity rather than enhanced recruitment.

\section{Discussion}

The discovery in 1998 that RANKL is the key osteoclastogenic cytokine positioned it as a principal anti-bone resorptive target (2). In fact, arrest of RANK signaling has proven effective treatment for postmenopausal osteoporosis (16). Thus, attention has turned to modulating RANKL's consequent intracellular signals as a reasonable therapeutic strategy.
A TRAF6-FLAG
RANK-HA
IP: anti-FLAG
IB: anti-HA
Western:
anti-HA
Western:
anti-FLAG

B FHL2-FLAG RANK-HA IP: anti-FLAG
IB: anti-HA Western: anti-HA Western: anti-FLAG

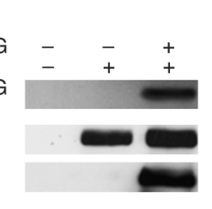

\section{C}
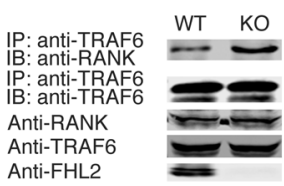

Anti-FHL2

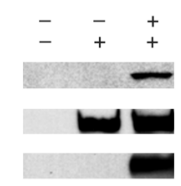

\section{Figure 5}

FHL2 inhibits TRAF6/RANK association in osteoclasts. FLAG-tagged TRAF6, FLAG-tagged FHL2, and HA-tagged RANK were cotransfected, in various combinations, into 293T cells. Protein expression was determined by anti-FLAG or anti-HA antibodies. Anti-FLAG antibody was used for immunoprecipitation and anti-HA for immunoblotting. (A) TRAF6 interacts with RANK. (B) FHL2 interacts with RANK. (C) After 4 days of exposure to M-CSF and RANKL, WT and $\mathrm{FHL}^{-1-}$ cells were lysed. Equal amounts of lysate were immunoprecipitated with antiTRAF6 antibody and the immunoprecipitate blotted with anti-RANK or anti-TRAF6 antibodies. Total cell lysate content of RANK, TRAF6, and FHL2 were determined by immunoblot. 
A

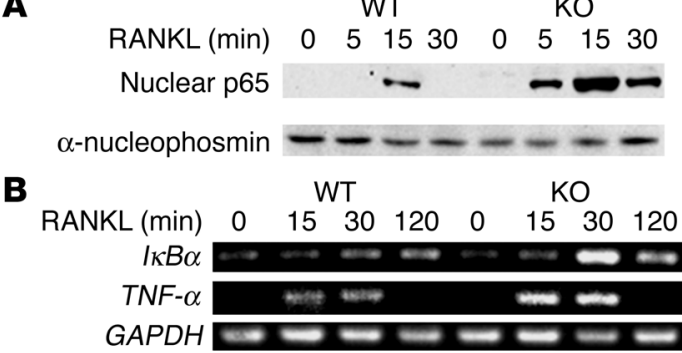

C

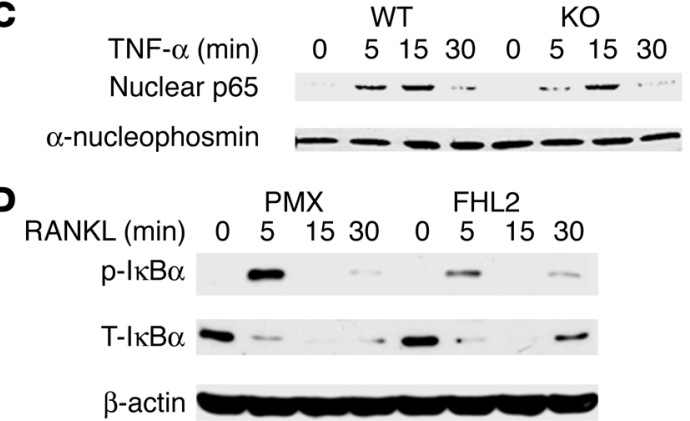

\section{Figure 6}

FHL2 modulates NF-KB activity. WT and FHL2-/- BMMs were cultured with M-CSF and RANKL. On day 3 , the cells were starved for 3 hours and then stimulated with RANKL $(100 \mathrm{ng} / \mathrm{ml})$ for up to 2 hours. (A) Nuclear proteins were extracted and NF-kB subunit p65 content was determined by immunoblot. $\alpha$-Nucleophosmin served as loading control. (B) $/ \kappa B \alpha$ and TNF- $\alpha$ mRNA expression was determined by RT-PCR. GAPDH mRNA served as loading control. (C) WT and FHL2 ${ }^{-/-}$BMMs were cultured with M-CSF and RANKL. On day 3, the cells were starved for 3 hours and then stimulated with TNF- $\alpha(10 \mathrm{ng} / \mathrm{ml})$ at $0,5,15$, and 30 minutes. Nuclear proteins were extracted, and NF- $\mathrm{KB}$ subunit p65 content was determined by immunoblot. $\alpha$-Nucleophosmin served as loading control. (D) WT BMMs were retrovirally transduced with empty vector (PMX) or vector expressing FHL2 and selected with blasticidin S, hydrochloride for 3 days. Selected cells were starved for 3 hours and stimulated with RANKL (100 ng/ml) for up to 30 minutes. Phosphorylated (p) and total (T) I $\mathrm{kB} \alpha$ were determined by immunoblot. $\beta$-actin served as loading control.

RANKL's receptor, RANK, is a component of the TNF receptor superfamily and, like all other members, has a cytoplasmic domain that associates with TRAFs (17). These adaptor molecules regulate cytokine signaling by linking TNF family receptors with downstream protein kinases, ubiquitin ligases, and other effector proteins (17). A total of 6 mammalian TRAFs are in hand, and all but TRAF4 recognize RANK. RANK binds TRAFs 1, 2, 3, and 5 at a multiple TRAF-binding site in its carboxyl terminal region (18). In contrast, TRAF6 targets a separate region of RANK, a domain essential for RANKL's effect on the osteoclast (19).

We initiated this study with the aim of identifying cellular proteins that associate with TRAF4. Our rationale for this undertaking was the fact that, while TRAF4 is not known to bind any recognized receptor, mice deficient in the protein have skeletal malformations (20). Thus, our aim was to begin to delineate the means by which TRAF4 regulates the skeleton by detailing molecules to which it complexes. To this end, we turned to a yeast 2-hybrid assay and discovered that TRAF4 associates with the LIM domain-only protein, FHL2.

LIM domain proteins typically serve as adaptors and scaffolds for multimolecular complexes $(21,22)$. They contain various combinations of 2 tandemly repeated zinc fingers with which they recognize their binding partners. FHL2 belongs to a subgroup of 5 homologous LIM domain-only proteins consisting exclusively of FHLs (23). It has been linked with tumorigenesis, cell differentiation, and nuclear receptor regulation (24). The protein, while originally believed expressed exclusively in heart (23), is also found in striated muscle (25) and, relevant to the present paper, in a myeloid cell line (26). FHL2 is localized within both the cytoplasm and the nucleus and shuttles between these compartments (24). In the nucleus, FHL2 serves as a cell and promoter-specific transcriptional coactivator or corepressor through its interaction with transcription factors such as the androgen receptor (27), activator protein-1 (28), cAMP response element binding protein (CREB) and CREM (29), and $\beta$-catenin $(30,31)$.

Each TRAF contains a TRAF domain, the carboxyl terminal of which mediates protein/protein interactions (5). Because the
TRAF domains of TRAF4 and TRAF6 diverge from those of the other 4 family members, we reasoned they would recognize distinct partners. Indeed, TRAF6, via its TRAF domain, binds FHL2 and does so with apparently greater efficiency relative to expressed protein than either TRAF2 or TRAF4. This selectivity of FHL2 by TRAF 6 and the central role this latter adaptor protein plays in osteoclast biology prompted us to focus on the interaction between the 2 molecules and how they, in combination, regulate the osteoclast. In fact, FHL2, specific among its subfamily of LIM domain-only proteins, increases pari passu with osteoclast differentiation. FHL2 also binds and colocalizes with TRAF6 in the differentiated cell and dose dependently inhibits its association with RANK. Given the importance of TRAF6 in RANK signaling, these observations suggested that FHL2 may have an impact on the osteoclast. To determine whether this is so, we turned to FHL2 $2^{-1-}$ mice and discovered that absence of the protein accelerate osteoclast differentiation. We also found that apoptotic changes appear in $\mathrm{FHL2}^{-{ }^{--}}$osteoclasts earlier than in their WT counterparts. Two possible explanations for the accelerated death of these mutant cells present themselves. In the first instance, RANKL induces apoptosis of osteoclast-like cells through TRAF6 (32), whose association with RANK is blunted by FHL2. Second, osteoclasts are terminally differentiated and incapable of mitotic activity. Because the absence of FHL2 prompts the cell to rapidly reach its ultimate state of differentiation, the polykaryon will also die earlier. This combination of accelerated differentiation and early death suggests that the longevity of FHL2-/- osteoclasts mirrors that of WT cells, which is also consonant with the similarity of osteoclast numbers in both genotypes in vivo.

NF- $\mathrm{KB}$ activation is an essential component of the osteoclastogenic process (33). Consistent with accelerated osteoclast precursor differentiation, nuclear translocation of the $\mathrm{p} 65$ subunit of the transcription complex is enhanced in FHL2-deficient osteoclast precursors. Moreover, FHL2 overexpression in osteoclasts blunts RANKL-stimulated IאB $\alpha$ phosphorylation. Thus, arrest of TRAF6mediated NF- $\kappa \mathrm{B}$ activation is likely to be an essential component of the antiosteoclastogenic properties of FHL2. 


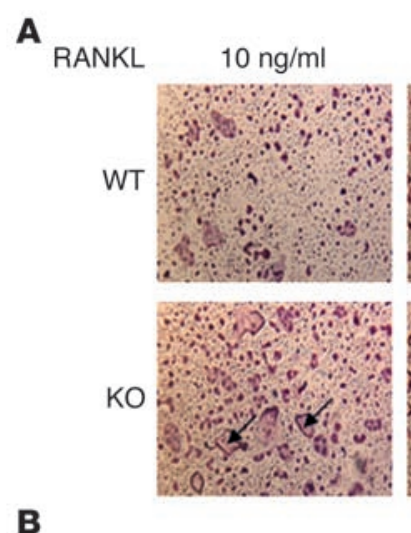

B

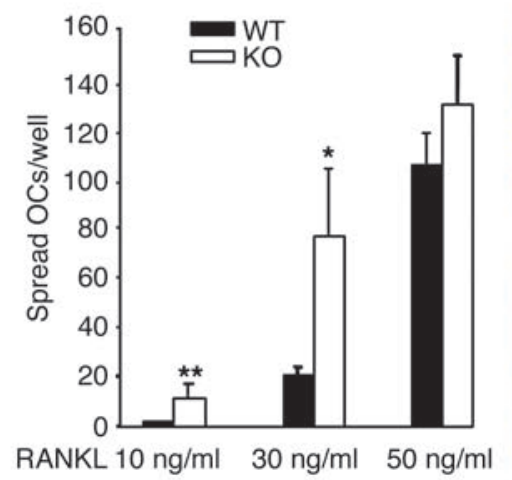

\section{$30 \mathrm{ng} / \mathrm{ml}$}

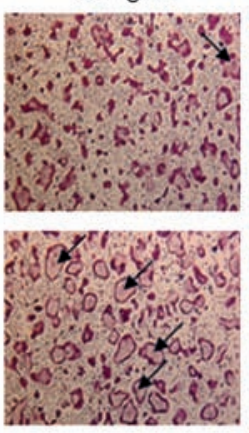

Spread

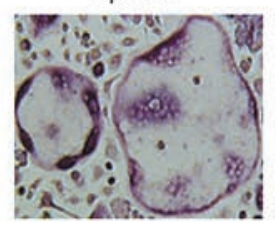

Nonspread

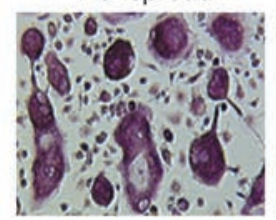

C

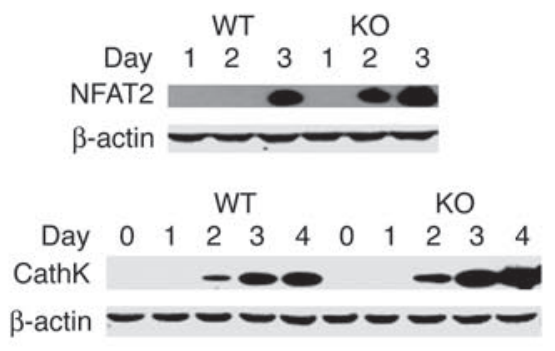

D
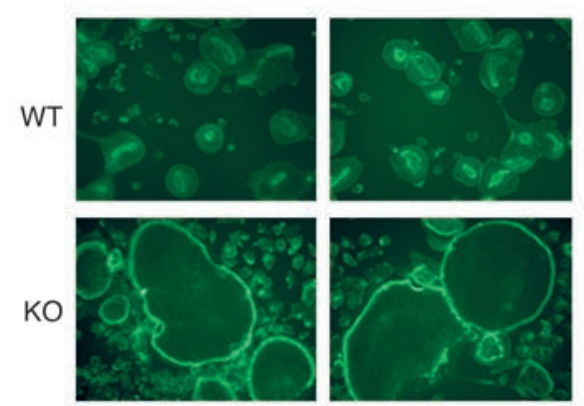

\section{Figure 7}

Absence of FHL2 accelerates osteoclast differentiation and cytoskeletal organization. (A) BMMs derived from WT and FHL2 $2^{-/-}$mice were maintained for 4 days in M-CSF and increasing concentrations of RANKL. The cells were then stained for TRAP activity to determine the relative numbers of characteristic spread osteoclasts (arrows). (B) Quantitative data derived from experiment detailed in A comparing the numbers of characteristic spread osteoclasts (OCs) as a function of RANKL concentration. Inset shows characteristic spread and nonspread osteoclasts, respectively. ${ }^{*} P<0.05$; ${ }^{\star \star} P<0.01$. (C) BMMs derived from WT and $F H L 2^{-/-}$mice were maintained for up to 4 days in M-CSF plus RANKL. Equal amounts of lysate were immunoblotted for NFAT2 and cathepsin K (CathK) content. $\beta$-actin serves as loading control. (D) WT and FHL2 ${ }^{-/}$BMMs were cultured on dentin with M-CSF and RANKL. On day 4, the cells were fixed and actin ring formation was assessed by rhodamine-phalloidin staining. Magnification: $\times 200(\mathbf{A})$ and $\times 600$ (B and D).

Despite the central role enjoyed by TRAF6 in regulating the osteoclast, its precise impact on the resorptive cell is controversial because of the varied phenotypes of mice lacking the adaptor protein. As expected, $\mathrm{TRAF6}^{-/}$mice are osteopetrotic regardless of strain and one group posits that their failure to resorb bone represents an absence of osteoclasts (9). Alternatively, another laboratory claims that TRAF6-deficient osteoclasts are generated but are dysfunctional, as they do not attach to bone, a likely cytoskeletal defect (8). While this discrepancy remains to be resolved, it may relate to the fact that RANKL has an impact not only on commitment of macrophages to the osteoclast phenotype but also on activation of mature osteoclasts. In this regard, Armstrong et al. report that TRAF6 is essential for osteoclast cytoskeletal organization and resorptive function but that partial redundancy exists among the various TRAFs in RANKL-mediated osteoclast differentiation (34). In fact, FHL2 has an impact on the osteoclast cytoskeleton as evidenced by either its deletion or excess. Specifically, osteoclasts lacking FHL2 have accelerated formation of large actin rings. This cytoskeletal structure isolates the resorptive microenvironment from the general extracellular space and is essential for optimal bone degradation, as it is absent in disorders of osteoclast function such as that characterizing deletion of the $\beta 3$ integrin (35). In this regard, FHL2 expression by osteoclasts is similar to that of the $\beta 3$ integrin by endothelial cells (36-38). Both proteins are abundant in culture but absent in naive cells in vivo, only to appear in states of cellular activation. These observations underscore the likelihood that discrepancies between gene expression in vitro and in naive animals represent a global activating effect of culture. Such a circumstance is also evident as regards the osteoclastogenic capacity of NF-кB-inducing kinase-deficient mice (14).

Similar to other molecules, such as SHIP-1 (39), which physiologically restrain osteoclast function, deletion of FHL2 results in exaggerated cytoskeletal organization leading to hyperresorptive osteoclasts and, in vivo, a deficit of bone mass following RANKL treatment. In contrast, yet also in keeping with inhibition of TRAF6-mediated differentiation and cytoskeletal organization, FHL2 overexpression desensitizes osteoclast precursors to RANKL, consequently limiting their differentiation and the ability of the polykaryons to spread effectively.

These studies expand our knowledge of the RANK signaling pathway and underscore the importance of TRAF6-associated proteins in regulating the osteoclast. Finally, FHL2 joins a growing collection of molecules not suspected of participating in skeletal biology that ultimately prove to be modulators of bone cell function and thus potential therapeutic targets, particularly in states of accelerated resorption. 

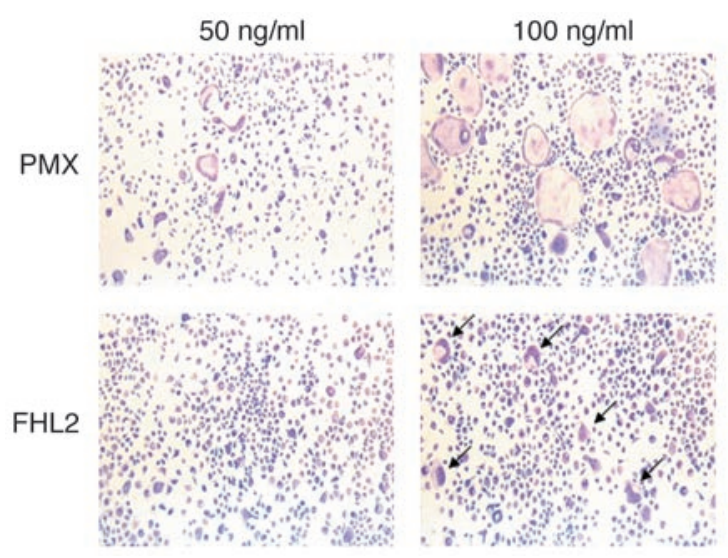

$200 \mathrm{ng} / \mathrm{ml}$

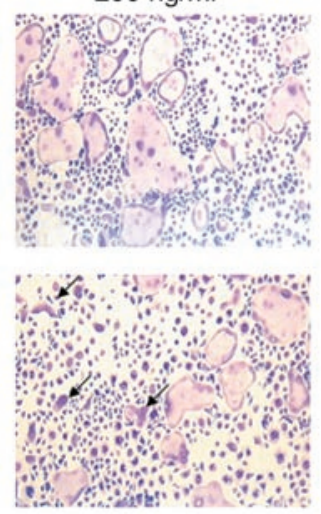

Figure 8

FHL2 overexpression attenuates osteoclast differentiation and spreading. BMMs were retrovirally transduced with empty vector or vector expressing FHL2 and selected with blasticidin S, hydrochloride for 3 days. Selected cells were stimulated with M-CSF and increasing doses of RANKL for 4 days after which the cultures were stained for TRAP activity. Arrows point to nonspread osteoclasts. Magnification: $\times 400$.

\section{Methods}

Mice. FHL2 ${ }^{-/-}$and WT mice on the hybrid Black Swiss-129-SV/J background were the products of heterozygous crosses (40). Animals were housed in the animal care unit of the Department of Pathology, Washington University School of Medicine, and were maintained according to guidelines of the Association for Assessment and Accreditation of Laboratory Animal Care. All animal experimentation was approved by the Animal Studies Committee of Washington University School of Medicine.

Reagents. Recombinant murine M-CSF was from R\&D Systems. Murine glutathione-S-transferase-RANKL was expressed in our laboratory as described previously (4). Anti-HA antibody was obtained from Covance Research Products. Anti-TRAF6 and anti-NFAT2 antibodies were obtained from Santa Cruz Biotechnology Inc. Antiactivated and anti-total caspase 3 antibodies were obtained from Cell Signaling Technology. All other chemicals were obtained from Sigma-Aldrich.

Two-hybrid library screening. A full-length TRAF4 coding sequence was cloned into pGBT9 (BD Biosciences - Clontech) to generate the pGBT9/ TRAF4 bait plasmid. The mouse embryo pACT2 cDNA library (a generous gift from Xu Cao, University of Alabama at Birmingham, Birmingham, Alabama, USA) was screened according to the manufacturer's instructions (BD Biosciences - Clontech).

Plasmids. TRAF2, TRAF4, and TRAF6 cDNA were obtained by RT-PCR and PCR from a murine primary osteoblast cDNA library generated in our laboratory. All TRAFs were coupled to FLAG tag (Sigma-Aldrich) at the C terminal in PcDNA3 vector (Invitrogen Corp.). RANK-HA plasmid was generated in our laboratory. FHL2 cDNA was recovered from the yeast 2-hybrid assay and was linked with either FLAG or HA at $\mathrm{C}$ terminal in PcDNA3 vector.

Immunoprecipitation and Western blot. FLAG-tagged full-length and truncated TRAF6, FLAG tagged FHL2, and HA-tagged full-length and truncated FHL2 cDNAs were subcloned into the mammalian expression vector pcDNA3 (Invitrogen Corp.). 293T cells were transfected with expression constructs using Fugene 6 as the transfection reagent according to the manufacturer's instructions (Promega). Cells were lysed 48 hours after transfection, and lysates were immunoprecipitated and immunoblotted with anti-FLAG or anti-HA antiserum. Anti-TRAF6, anti-FHL2, and anti-RANK antibodies were used to detect endogenous protein-protein interaction. Nuclear p 65 translocation experiments were performed by using lysates of preosteoclasts generated by exposure of BMMs to M-CSF and RANKL for 3 days.

Immunofluorescence. Intracellular TRAF6 and FHL2 were labeled with fluorescent markers as described (41). In brief, cells were fixed with $3 \%$ paraformaldehyde in PBS for 20 minutes. Free aldehyde groups were quenched with $50 \mathrm{mM} \mathrm{NH}_{4} \mathrm{Cl}$ in PBS for 10 minutes. Nonspecific binding was blocked by incubation in PBS containing $0.2 \%$ bovine serum albumin and $0.05 \%$ saponin (PBSBS) for 15 minutes. The cells were then incubated with primary antibodies in PBSBS for 1 hour. Primary antibody binding was visualized using fluorescent dye-conjugated secondary antibodies (Jackson ImmunoResearch Laboratories Inc.) in PBSBS for 45 minutes. Immunofluorescence analysis was performed using a Nikon fluorescence microscope equipped with a CCD camera. Primary antibodies used include rabbit anti-FLAG (Sigma-Aldrich), monoclonal anti-HA (Covance Research Products), monoclonal anti-FHL2, and polyclonal anti-TRAF6 (Santa Cruz Biotechnology Inc.).

Primary cells and cell lines. Macrophages/osteoclast precursors and osteoclasts were generated from bone marrow precursors as described (42). C2C12 and 293 T cells were obtained from ATCC and were maintained as described.

Characterization of osteoclasts. BMMs were cultured in 96-well cell culture dishes in the presence of M-CSF $(10 \mathrm{ng} / \mathrm{ml})$ and RANKL $(100 \mathrm{ng} / \mathrm{ml}$ or
A

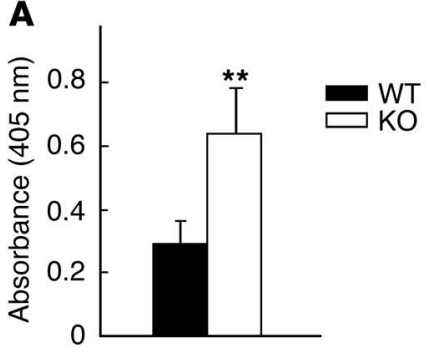

B

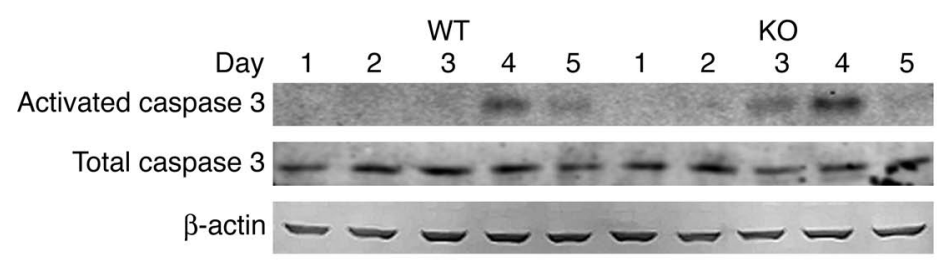

Figure 9

FHL2-deficient osteoclasts undergo early apoptosis. WT and FHL2-/- BMMs were cultured in M-CSF and RANKL. (A) The magnitude of apoptosis was measured by ELISA 4 days later. ${ }^{* *} P<0.01$. (B) Activated and total caspase 3 were measured by immunoblot with time. $\beta$-actin served as loading control. 
A

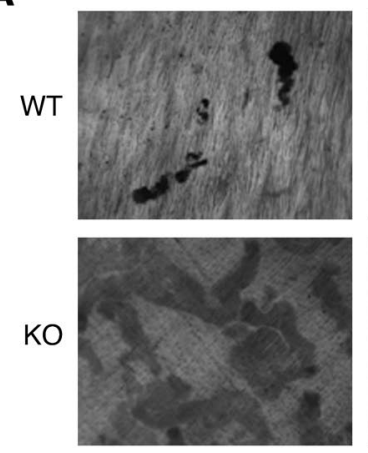

B

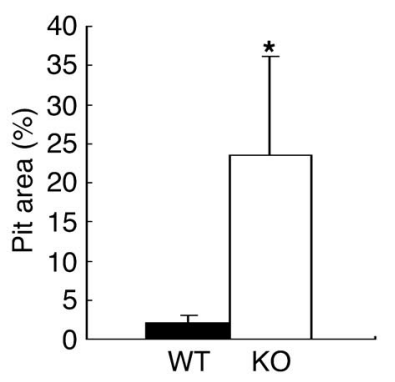

C

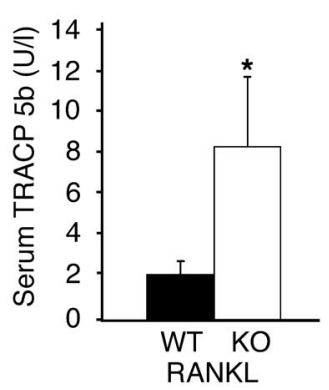

D

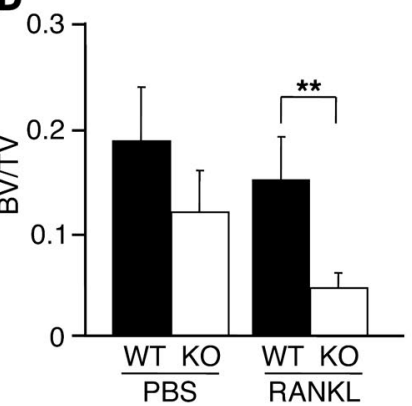

Figure 10

Absence of FHL2 enhances osteoclast resorptive activity and RANKL-stimulated bone loss. (A) WT and $\mathrm{FHL2}^{-1-}$ BMMs were cultured on dentin with M-CSF and RANKL. Resorption lacuna formation was examined on day 4 by Coomassie brilliant blue staining. Magnification: $\times 200$. (B) Quantitative analysis of experiment detailed in A. Pit area (\%), percentage of dentin surface occupied by resorption lacunae. (C) WT and $\mathrm{FHL2}^{-/-}$mice were administered $100 \mu \mathrm{g}$ RANKL subcutaneously for 7 days. Serum TRACP $5 b$ levels were determined by ELISA. (D) WT and FHL2--- mice were administered PBS (control) or RANKL $(100 \mu \mathrm{g})$ daily for 7 days. The tibiae were recovered on day 9 and trabecular bone volume (BV/TV) determined by micro-CT. ${ }^{*} P<0.05 ;{ }^{*} P<0.01$. doses detailed in figure legends). Media were changed on day 3. Osteoclast-like cells were characterized by staining for tartrate-resistant acid phosphatase (TRAP) activity. The number of osteoclasts was counted as described previously (42). The characteristics of spread as compared to nonspread osteoclasts are illustrated in Figure 7B.

RNA extraction and amplification by RT-PCR. BMMs were exposed to $\mathrm{M}-\mathrm{CSF}$ and RANKL for various times as detailed in figure legends. RNA purification and RT-PCR conditions were as described previously (42). The oligonucleotide primers used are as follows: FHL1, 5'-GTTTCACCGCTGTGGAGGAC, 3 '-GAGGGAGGGAGGGAGCAAAG; FHL2, 5'-CCCCATTAGTGGTCTGGGTG, 3'-CCAGGGCAGCAAGAGGAAGC; FHL3, 5'-GCGCCCCATCGTAGGACTCG, 3' - CGTTCTAGGGAGGGGTTGGG; FHL4, 5' - -GGGTG CAAGAACCCCATCAC, 3'-GGAGCAGCTTCACAGAGGTG; ACT, 5' GCCTGCTGTGCACAGACTGC, 3'-GGGTTTGGTGCAGGCTGCAC.

Bone resorption. Osteoclasts were generated on whale dentin slices from BMMs under conditions described above. After 4 days the cells were stained for TRAP activity. For actin-ring staining, cells were fixed in $4 \%$ paraformaldehyde, permeabilized in $0.1 \%$ Triton X-100, rinsed in PBS, and immunostained with Alexa 488 phalloidin (Invitrogen Corp.). To quantitate resorption lacunae, cells were removed from the dentin slices with

\section{Figure 11}

FHL2 is expressed only in activated osteoclasts. (A) WT mice were administered PBS or RANKL (100 $\mu \mathrm{g} /$ day) for 7 days. On day 9 , histological sections of tibiae were stained for TRAP activity (red reaction product) or immunostained using an anti-FHL2 mAb. Osteoclasts in the RANKL-treated, but not PBS-treated, mice stained positive for the brown reaction product. (B) Serum transfer inflammatory arthritis was induced in WT mice. After 7 days, histological sections of the inflamed joints were immunostained for FHL-2, which is present in osteoclasts. (C) WT and $\mathrm{FHL2}^{-1-}$ mice were administered arthrogenic serum to induce inflammatory arthritis or PBS as control. Serum TRACP $5 b$ levels were measured at days 0,7 , and 14 . ${ }^{\star} P<0.05$ vs. WT serum. Magnification: $\times 400$.
$0.5 \mathrm{M}$ ammonium hydroxide and mechanical agitation. Dentin slides were stained with Coomassie brilliant blue.

A
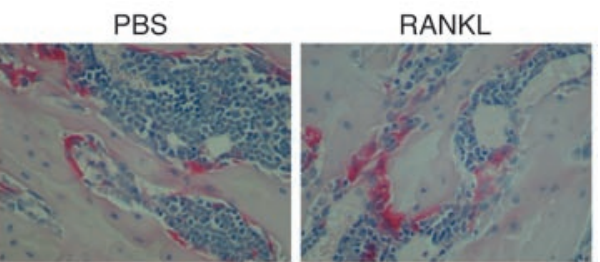

FHL2
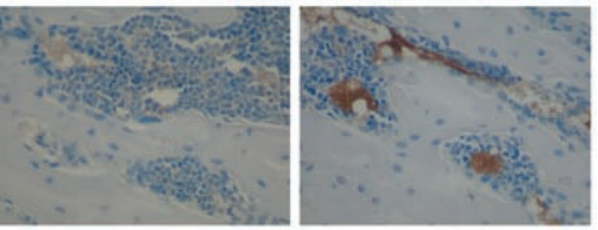

B

Serum-induced inflammatory arthritis

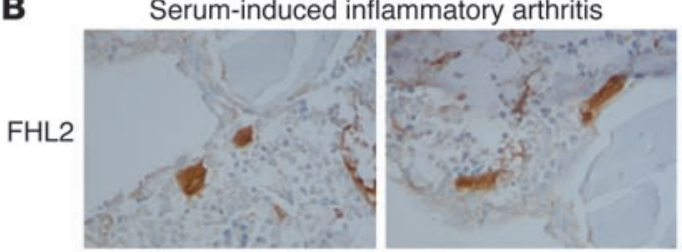

C

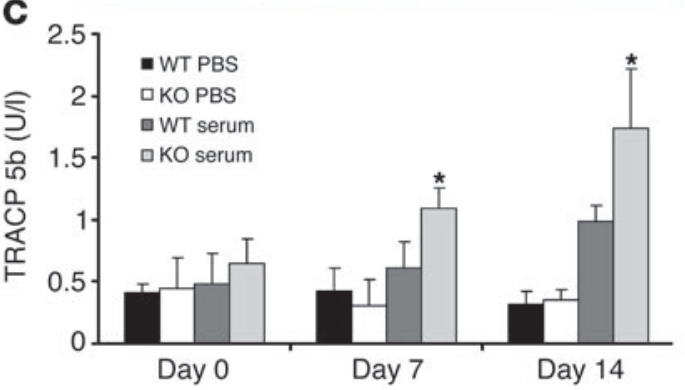


Cell stimulation and immunoblotting. For NF- $\mathrm{KB}$ signaling experiments, primary macrophages were infected with empty vector or FHL2-expressing retrovirus (43). Infected cells were selected by blasticidin S, hydrochloride (BSR) for 3 days and starved for 3 hours. RANKL $(100 \mathrm{ng} / \mathrm{ml})$ was added to the media, cells were lysed at $0,5,15$, and 30 minutes, and the lysate was subjected to immunoblotting.

RANKL-stimulated bone loss in vivo. RANKL (100 $\mu \mathrm{g}$ per mouse per day) or PBS, as control, was injected subcutaneously once per day for 7 days. Two days after the final injection, mice were sacrificed by $\mathrm{CO}_{2}$ narcosis and the tibias, devoid of soft tissue, were used to determine trabecular bone volume by micro-CT.

Cell death detection assay. Cell death detection assay was performed according to the protocol of the Cell Death Detection ELISA kit (Roche Diagnostics Corp.). In brief, WT and FHL2 $-/$ (KO) BMMs were cultured in 48-well plates $\left(1.5 \times 10^{4}\right.$ cells $/$ well $)$ in M-CSF and RANKL $(100 \mathrm{ng} / \mathrm{ml})$ for 4 days. On days 4 and 5, cells were lysated and centrifuged, respectively, at $200 \mathrm{~g}$ for 10 minutes. Supernants $(20 \mu \mathrm{l})$ were mixed with $80 \mu \mathrm{l}$ immunoreagent for 2 hours with shaking at room temperature. The solution was aspirated, and the wells were washed 3 times with $300 \mathrm{ml}$ incubation buffer. Substrate solution $(100 \mu \mathrm{l})$ was added to each well and incubated on a plate shaker at $250 \mathrm{rpm}$ until color development was complete. The absorbance was read at $405 \mathrm{~nm}$.

Immunohistochemical staining. The PBS- and RANKL-injected FHL2 WT and FHL2 $2^{-/}$mice trabecular bone tissues were paraffin embedded and cut into 5 - $\mu \mathrm{m}$ sections. Samples were briefly treated with hydrogen peroxide to block endogenous peroxidase. Antigen retrieval method was used. To lessen nonspecific binding, the samples were blocked with a mouse Ig reagent. Monoclonal FHL2 antibody was diluted 1:100 and incubated at $4{ }^{\circ} \mathrm{C}$ overnight, followed by incubation with biotinylated anti-mouse second antibody using a Vectastain Elite ABC kit (Vector Laboratories) according to the manufacturer's protocol. The samples were developed with diaminobenzidine peroxidase substrate and coun- terstained with hematoxylin. The samples were mounted and coverslipped. As another negative control, the FHL2 WT samples injected with either PBS or RANKL were without primary antibody but using secondary biotinylated.

Inflammatory arthritis model and serum TRACP $5 b$ assay. Serum transfer inflammatory arthritis was induced as described (44). To determine bone resorptive activity in vivo, RANKL $(100 \mu \mathrm{g} /$ mouse/day) or PBS was injected subcutaneously once per day for 7 days. Two days after the final injection, serum was collected. TRACP $5 \mathrm{~b}$ concentration was determined according to the protocol of the Mouse TRAP Assay kit (Immunodiagnostic Systems Ltd.).

Statistics. Data are expressed as mean \pm SD. Statistical significance was determined by 2 -tailed Student's $t$ test. $P<0.05$ was considered statistically significant.

\section{Acknowledgments}

This study was supported by NIH grants AR46852 and AR48812 (F.P. Ross), AR32788, AR46523, AR48853, and DK-56341 (Clinical Nutrition Research Unit, Washington University School of Medicine) (S.L. Teitelbaum). The authors wish to thank Crystal Idleburg for her assistance with selected experiments and Paulette Shubert for preparing this manuscript.

Received for publication March 2, 2005, and accepted in revised form July 12, 2005.

Address correspondence to: Steven L. Teitelbaum, Washington University School of Medicine, Department of Pathology, Campus Box 8118, 660 South Euclid Avenue, St. Louis, Missouri 63110, USA. Phone: (314) 454-8463; Fax: (314) 454-5505; E-mail: teitelbs@wustl.edu.
1. Teitelbaum, S.L. 2000. Bone resorption by osteoclasts. Science. 289:1504-1508.

2. Lacey, D.L., et al. 1998. Osteoprotegerin ligand is a cytokine that regulates osteoclast differentiation and activation. Cell. 93:165-176.

3. Dougall, W.C., et al. 1999. RANK is essential for osteoclast and lymph node development. Genes Dev. 13:2412-2424

4. Lam, J., Nelson, C.A., Ross, F.P., Teitelbaum, S.L., and Fremont, D.L. 2001. Crystal structure of TRANCE/RANKL cytokine reveals determinants of receptor-ligand specificity. J. Clin. Invest. 108:971-980. doi:10.1172/JCI200113890.

5. Bradley, J.R., and Pober, J.S. 2001. Tumor necrosis factor receptor-associated factors (TRAFs). Oncogene. 20:6482-6491.

6. Cao, Z., Xiong, J., Takeuchi, M., Kurama, T., and Goeddel, D.V. 1996. TRAF6 is a signal transducer for interleukin-1. Nature. 383:443-446.

7. Ye, H., et al. 2002. Distinct molecular mechanism for initiating TRAF6 signalling. Nature. 418:443-447.

8. Lomaga, M.A., et al. 1999. TRAF6 deficiency results in osteopetrosis and defective interleukin-1, CD40, and LPS signaling. Genes Dev. 13:1015-1024.

9. Naito, A., et al. 1999. Severe osteopetrosis, defective interleukin-1 signalling and lymph node organogenesis in TRAF6-deficient mice. Genes Cells. 4:353-362.

10. Kadrmas, J.L., and Beckerle, M.C. 2004. The LIM domain: from the cytoskeleton to the nucleus. Nat. Rev. Mol. Cell Biol. 5:920-931.

11. Morgan, M.J., and Whawell, S.A. 2000. The structure of the human LIM protein ACT gene and its expression in tumor cell lines. Biochem. Biophys. Res. Commun. 273:776-783.

12. Kobayashi, N., et al. 2001. Segregation of TRAF6mediated signaling pathways clarifies its role in osteoclastogenesis. EMBO J. 20:1271-1280.
13. Samson, T., et al. 2004. The LIM-only proteins FHL2 and FHL 3 interact with $\alpha$ - and $\beta$-subunits of the muscle $\alpha 7 \beta 1$ integrin receptor. J. Biol. Chem. 279:28641-28652.

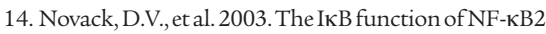
p100 controls stimulated osteoclastogenesis. J. Exp. Med. 198:771-781.

15. Kitaura, H., et al. 2003. Marrow stromal cells and osteoclast precursors differentially contribute to TNF- $\alpha$ induced osteoclastogenesis in vivo.J.Immunol. 173:4838-4846.

16. Becker, P.J., et al. 2004, A single-dose placebo-controlled study of AMG 162, a fully human monoclonal antibody to RANKL, in post-menopausal women. J. Bone Miner. Res. 19:1059-1066.

17. Inoue, J., et al. 2000. Tumor necrosis factor receptor-associated factor (TRAF) family: adapter proteins that mediate cytokine signaling. Exp. Cell Res. 254:14-24.

18. Arch, R.H., Gedrich, R.S., and Thompson, C.B 1998. Tumor necrosis factor receptor-associated factors (TRAFs)-a family of adapter proteins that regulates life and death. Genes Dev. 12:2821-2830.

19. Wu, H., and Arron, J.R. 2003. TRAF6, a molecular bridge spanning adaptive immunity, innate immunity and osteoimmunology. Bioessays. 25:1096-1105.

20. Regnier, C.H., et al. 2002. Impaired neural tube closure, axial skeleton malformations, and tracheal ring disruption in TRAF4-deficient mice. Proc. Natl. Acad. Sci. U. S. A. 99:5585-5590.

21. Dawid, I.B., Breen, J.J., and Toyama, R. 1998. LIM domains: multiple roles as adapters and functional modifiers in protein interactions. Trends Genet. 14:156-162.

22. Bach, I. 2000. The LIM domain: regulation by association. Mech. Dev. 91:5-17.

23. Chan, K.K., et al. 1998. Molecular cloning and characterization of FHL2, a novel LIM domain protein preferentially expressed in human heart. Gene. 210:345-350.

24. Muller, J.M., et al. 2002. The transcriptional coactivator FHL2 transmits Rho signals from the cell membrane into the nucleus. EMBO J. 21:736-748.

25. Chu, P.-H., Ruiz-Lozano, P., Zhou, Q., Cai, C., and Chen, J. 2000. Expression patterns of FHL/SLIM family members suggest important functional roles in skeletal muscle and cardiovascular system. Mech. Dev. 95:259-265.

26. McLoughlin, P., et al. 2002. The LIM-only protein DRAL/FHL2 interacts with and is a corepressor for the promyelocytic leukemia zinc finger protein. J. Biol. Chem. 277:37045-37053.

27. Muller, J.M., et al. 2000. FHL2, a novel tissue-specific coactivator of the androgen receptor. EMBOJ. 19:359-369.

28. Morlon, A., and Sassone-Corsi, P. 2003. The LIMonly protein FHL2 is a serum-inducible transcriptional coactivator of AP-1. Proc. Natl. Acad. Sci. U. S. A. 100:3977-3982.

29. Fimia, G.M., De Cesare, D., and Sassone-Corsi, P. 2000. A family of LIM-only transcriptional coactivators: tissue-specific expression and selective activation of CREB and CREM. Mol. Cell. Biol. 20:8613-8622.

30. Wei, Y., et al. 2003. Identification of the LIM protein FHL2 as a coactivator of $\beta$-catenin. J. Biol. Chem. 278:5188-5194.

31. Labalette, C., Renard, C.-A., Neuveut, C., Buendia, M.-A., and Wei, Y. 2004. Interaction and functional cooperation between the LIM protein FHL2, CBP/p300, and $\beta$-catenin. Mol. Cell. Biol. 24:10689-10702.

32. Bharti, A.C., Takada, Y., Shishodia, S., and Aggarwal, B.B. 2004. Evidence that receptor activator of 
nuclear factor (NF)- $\kappa$ B ligand can suppress cell proliferation and induce apoptosis through activation of a NF-KB-independent and TRAF6-dependent mechanism. J. Biol. Chem. 279:6065-6076.

33. Rothwarf, D.M., and Karin, M. 1999. The NF-кB activation pathway: a paradigm in information transfer from membrane to nucleus [review]. Sci. STKE. 5:re1.

34. Armstrong, A.P., et al. 2002. A RANK/TRAF6dependent signal transduction pathway is essential for osteoclast cytoskeletal organization and resorptive function. J. Biol. Chem. 277:44347-44356.

35. McHugh, K.P., et al. 2000. Mice lacking $\beta 3$ integrins are osteosclerotic because of dysfunctional osteoclasts. J. Clin. Invest. 105:433-440.

36. Stupack, D.G., and Cheresh, D.A. 2004. Integrins and angiogenesis. In Current topics in developmental biology. Volume 64. G. Schatten, editor. Academic Press. San Diego, California, USA. 207-238.

37. Cheresh, D.A. 1987. Human endothelial cells synthesize and express an Arg-Gly-Asp- directed adhesion receptor involved in attachment to fibrinogen and von Willebrand factor. Proc. Natl. Acad. Sci.U. S. A. 87:6471-6475.

38. Okada, Y., et al. 1996. Integrin alphavbeta3 is expressed in selected microvessels after focal cerebral ischemia. Am. J. Pathol. 149:37-44.

39. Takeshita, S., et al. 2002. SHIP-deficient mice are severely osteoporotic due to increased numbers of hyper-resorptive osteoclasts. Nat. Med. 8:943-949.

40. Chu, P.-H., Bardwell, W.M., Gu, Y., Ross, J., Jr., and Chen, J. 2000. FHL2 (SLIM3) is not essential for cardiac development and function. Mol. Cell. Biol. 20:7460-7462.
41. Zhao, H., Laitala-Leinonen, T., Parikka, V., and Vaananen, H.K. 2001. Downregulation of small GTPase Rab7 impairs osteoclast polarization and bone resorption. J. Biol. Chem. 276:39295-39302.

42. Wang, M.W.-H., et al. 2004. The HIV protease inhibitor ritonavir blocks osteoclastogenesis and function by impairing RANKL-induced signaling. J. Clin. Invest. 114:206-213. doi:10.1172/ JCI200415797.

43. Faccio, R., Zallone, A., Ross, F.P., and Teitelbaum, S.L. 2003. c-Fms and the $\alpha v \beta 3$ integrin collaborate during osteoclast differentiation. J. Clin. Invest. 111:749-758. doi:10.1172/JCI200316924.

44. Aya, K., et al. 2005. NF-кB-inducing kinase controls lymphocyte and osteoclast activities in inflammatory arthritis. J. Clin. Invest. 115:1848-1854. doi:10.1172/JCI23763. 